\title{
Planning and real-time management of smart grids with high PV penetration in Italy
}

Lorenzo Bottaccioli PhD

Postdoctoral Researcher, Politecnico di Torino, Turin, Italy

Abouzar Estebsari PhD

Assistant Professor, Politecnico di Torino, Turin, Italy

Edoardo Patti PhD

Assistant Professor, Politecnico di Torino, Turin, Italy
Enrico Pons PhD

Assistant Professor, Politecnico di Torino, Turin, Italy (corresponding author: enrico.pons@polito.it) (Orcid:0000-0001-9391-4108)

Andrea Acquaviva PhD

Associate Professor, Politecnico di Torino, Turin, Italy

For planning and development and in real-time operation of smart grids, it is important to evaluate the impacts of photovoltaic (PV) distributed generation. This paper presents an integrated platform, constituted of two main components: a PV simulator and a real-time distribution network simulator. The first, designed and developed following the microservice approach and providing Representational State Transfer web services, simulates real-sky solar radiation on rooftops and estimates the PV energy production. The second, based on a digital real-time power systems simulator, simulates the behaviour of the electricity network under the simulated generation scenarios. The platform is tested on a case study based on real data for a district of the city of Turin, Italy. In the results, the authors show possible applications of the platform for power flow forecasting during real-time operation and for detecting possible voltage and transformers' capacity problems during planning due to high penetration of renewable energy sources. In particular, the results show that the case study distribution network, in the actual configuration, is not ready to accommodate all the generation capacity that can be installed, as, in certain hours of the day and on certain days of the year, the capacity of some transformers is exceeded.

\section{Introduction}

In recent years, governments have provided incentives for connecting to electricity networks low-carbon dioxide and sustainable generation technologies, in particular photovoltaic (PV) systems. The conversion of passive building rooftops into active PV rooftops is becoming more popular in all cities because in this way it is possible to exploit the available surfaces without subtracting additional portions of land from other uses. Recent works (Brito et al., 2017; Yu et al., 2017) have demonstrated the importance and availability of building facades for the deployment of PV systems in addition to classic rooftop installations. This possibility could provide a significant additional area for PV deployment, but should be carefully evaluated, as in old cities with narrow streets, PVs on facades would produce a small amount of energy and many old buildings with historical importance cannot accommodate PVs on their facades. The importance of integrating PV systems in buildings was highlighted by Varney and Vahdati (2014), where the authors studied possible integration of PV systems with heating and cooling systems in buildings, proving an increase in energy efficiency.

Currently, there is no regulation for distributed generation (DG) placement and there is no optimised planning strategy. For these reasons, specific tools for the analysis of possible scenarios are really important for different stakeholders, such as distribution system operators (DSOs), energy and city planners and policymakers. One of the critical issues relates to the power balance at the power transformers, which might not have sufficient spare capacity to accommodate the solar electricity feed-in (Freitas et al., 2018a).
Keane et al. (2013) and Spertino et al. (2014) stated that robust tools that help assess the capabilities and requirements of the networks are required for PV system deployment. In this assessment, it is not sufficient to consider the maximum installed peak power for PV production, but it is necessary to perform more sophisticated calculations, taking into account solar irradiance and demand hourly variation (Freitas et al., 2018a).

Geographic information system (GIS) tools have been widely used and are recognised as useful tools for renewable energy planning and estimation as reported by Domínguez and Amador (2007). In their work, Gagliano et al. (2017) proposed a GIS methodology for managing energy use at the urban scale. Freitas et al. (2015) reported how GIS tools have been applied for solar energy applications in urban contexts. Camargo et al. (2015) highlighted that available GIS tools for PV planning are limited because these neglect the time domain. Classic internet (web)-based platforms for PV potential analysis, such as those of Suri et al. (2008), Mapdwell (2018), de Sousa et al. (2012) and De Amicis et al. (2012), do not perform time-dependent simulations, but only yearly or monthly estimations. Furthermore, such services do not take into account electricity consumption and network topology, which is relevant to estimating realistic integration of PV systems into the grid.

Camargo et al. (2015), Jakubiec and Reinhart (2013), Luka et al. (2014) and Bottaccioli et al. (2017a) proposed new methodologies and solutions that integrate spatial and temporal domains in PV energy estimation. Their works have paved the way to new 
Engineering Sustainability

Volume 172 Issue ES6
Planning and real-time management

of smart grids with high PV penetration

in Italy

Bottaccioli, Estebsari, Patti, Pons and Acquaviva possibilities of spatio-temporal analysis in the assessment of PV potential. Spatio-temporal simulations enable the assessment of PV potential by integrating consumption and generation profiles with grid topology. In particular, Camargo et al. (2015) integrated simulated PV production with electricity consumption data for correct PV integration to avoid network congestions. Furthermore, Bottaccioli et al. (2017b) co-simulated in real time the integration of PV systems with storage systems to study battery management strategies, considering metered consumption data and GIS simulation of PV production.

In the methodology that the authors propose, a PV simulator is coupled with a real-time grid simulator, in order to be able not only to consider consumption data and network topology, but also to take into account the electric behaviour of the distribution system.

There are a lot of examples on the applications of real-time simulation to electrical systems including grids, power electronics and control systems. There are two main types of simulations: electromagnetic transient and phasor simulations; however, there are some examples of combinations of these. The most common commercial application of real-time simulation is in the prototyping stage of manufacturing a device or developing a system. This capability of real-time simulation provides the possibility of testing even when there are no physical prototypes (Bompard et al., 2016; Dufour et al., 2008; Vamsidhar and Fernandes, 2004). Real-time simulation is also widely used before and after prototyping as design and test phases, respectively - for example, in PV generation (Park and Yu, 2004) and wind conversion systems (Pak and Dinavahi, 2009; Wang and Gao, 2008).

The solution presented in this paper is devoted to satisfying the needs of different stakeholders: $(a)$ energy communities can use it to plan large PV system deployments and perform feasibility studies as proposed in the authors' previous research (Bottaccioli et al., 2015); (b) DSOs can simulate new control strategies for network balancing and plan retrofits and/or extensions of the existing distribution grid in planning phase and also better estimate network power flow during real-time operation; and (c) energy and city planners can evaluate the impacts of large PV system installations or monitor the performance of existing ones.

In the following sections, the software architecture is presented, with a brief description of the different modules. The proposed methodology and software architecture, which was previously presented by Bottaccioli et al. $(2017 \mathrm{c})$, is then applied to a district of the city of Turin, Italy, as a case study.

\section{Software architecture for planning PV system integration into smart grids}

This section describes the authors' proposed solution for planning PV system deployment in a smart-grid environment and evaluating its impact in the distribution network. As shown in Figure 1, the distributed software infrastructure consists of three

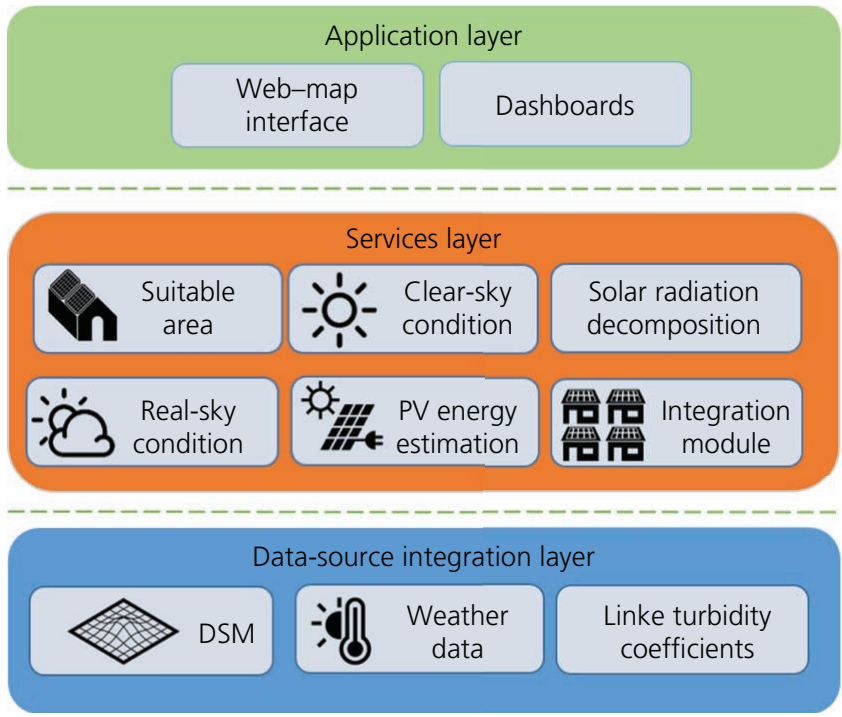

Figure 1. Scheme of the software architecture for PV systems integration into smart grids. DSM, digital surface model

layers: (a) data-source integration layer, (b) services layer and (c) application layer. Each layer is described in detail in the following. It is worth noting that the behaviour of a smart grid is simulated through a real-time grid simulator integrated in the architecture.

\subsection{PV system simulator}

In this work, the authors exploit the PV simulator presented in their previous work (Bottaccioli et al., 2016, 2017a) to estimate the energy generation profiles of PV systems in real-sky conditions. As shown in Figure 1, the PV simulator has been designed and developed following the microservice approach (Fowler and Lewis, 2014; Newman, 2015) and providing Representational State Transfer (Rest) web services (Fielding and Taylor, 2002). The main inputs of this simulator (see the datasource integration layer in Figure 1) are (a) a digital surface model (DSM) and (b) weather data - that is, trends of air temperature and global horizontal solar radiation (GHI). DSM is a high-resolution raster image. It represents the terrain elevation of buildings of interest. A DSM allows recognising encumbrances on rooftops (e.g. chimneys and dormer) that prevent the deployment of PV panels. In this work, the authors used a DSM resulting from some lidar flights.

The services layer consists of different software modules and estimates the PV energy production for a given urban area. The clear-sky condition module exploits the DSM to estimate the evolution of shadows in rooftops over the year, with $15 \mathrm{~min}$ intervals. As a result, this process produces a shadow model of the city and, together with the suitable area module, identifies the available areas on the roof where PV panels can be deployed. The evolution of irradiance on rooftops in real-sky conditions is computed starting from GHI trends retrieved from personal or 
third-party weather stations such as Weather Underground (2017). First, the solar radiation decomposition module decomposes GHI to estimate both direct normal incident radiation (DNI) and diffuse horizontal incident radiation (DHI) (Hofierka and Kaňuk, 2009), exploiting decomposition models in literature, such as those of Karatasou et al. (2003), Engerer (2015) and Ruiz-Arias et al. (2010). In decomposing GHI into DNI and DHI, the PV simulator also takes into account the attenuation caused by air pollution by applying the Linke turbidity coefficient (Linke, 1922). Then, the real-sky condition module combines DNI and DHI with the shadow model to estimate the irradiance on the available areas obtained in the previous step. The PV energy estimation module provides an estimation of the energy production by exploiting all the information computed in the previous steps and by applying the methodology presented by Brihmat and Mekhtoub (2013). The accuracy of estimating the energy production has already been demonstrated by Bottaccioli et al. (2017a). Finally, the authors extended the PV simulator with the integration module, which integrates and correlates information coming from the smart grid (e.g. energy consumption) with the estimated PV energy production data for the same geographic area. The integration module also enables communication with the real-time grid simulator (see Section 2.2). It is worth noting that this module has been designed to integrate also other software platforms for collecting real-time data from the smart grid, such as the platforms reported by Patti et al. $(2015,2016)$ and Pau et al. (2018).

Finally, the application layer is devoted to user applications, such as the web-map interface and dashboards, to provide information about performed simulations across the city.

\subsection{Real-time grid simulator}

Real-time simulation is a highly reliable method based on electromagnetic transient simulation which can emulate the realworld system. It provides very trustable real-like information on impacts and benefits of new strategies or devices, which could support decision-making from real-time operation and control phase to long-term planning. The possibility of ex ante tests reduces costs, and enables more complete and continuous testing of the entire system without interruption. Many possible configurations without physical modification can be also tested safely under possibly dangerous conditions.

Real-time simulation actually reproduces the behaviour of a physical system (e.g. electrical distribution grid) through running its computer-based model at the same rate as the actual clock time. In other words, in real-time simulation, when the simulation clock reaches a certain time (e.g. $1 \mathrm{~s})$, the same amount of time (1 s) has passed in the real world. It is typically used for highspeed simulations, closed-loop testing of protection and control equipment and generally all what-if analyses. Real-time simulation is actually simulating a system, which could realistically respond to its environment, when the inputs/outputs of the simulation are synchronous with the real world.

Considering the advantages of real-time simulation, there are a variety of applications to different domains such as electricity systems, mechatronics, robotics and industrial automation, automotive application, aerospace, all-electric ships and electric train networks, operator and technician training, electric drive and motor development and testing and power systems. Among all the mentioned fields, power systems are the main application domain of real-time simulation, using standard hardware-software configuration given by simulator providers. There are two main real-time simulation system providers: RTDS and Opal-RT: RTDS products are mainly for power system simulations, and about $80 \%$ of Opal-RT simulators are used for power system applications.

Regarding electricity systems, real-time simulation is widely used in protection and control system development and testing; DG modelling, in particular renewable energy source integration (e.g. PV generation penetration); and intelligent grid development.

The purpose of using a real-time simulator in this work is to model a realistic distribution network to support investigations in terms of PV penetration impacts in real-world situations. The objective is the simulation of the behaviour of prosumers and the set-up of a software-in-the-loop (SIL) platform for the laboratory validation of new control, operation and planning algorithms for smart-grid management (Estebsari et al., 2016).

As stated, the distribution grid needs to be first modelled for the specific application. The tool for implementing the modelled grid depends on the real-time simulation firmware configuration. The authors use Opal-RT with eMegasim configuration, which requires Matlab Simulink as the development environment. Therefore, the grid model is implemented in Matlab Simulink using Matlab toolbox blocks, the authors' developed blocks and some external special blocks provided by Opal-RT in an additional library. The interface between the development environment and simulator control is called RT Lab.

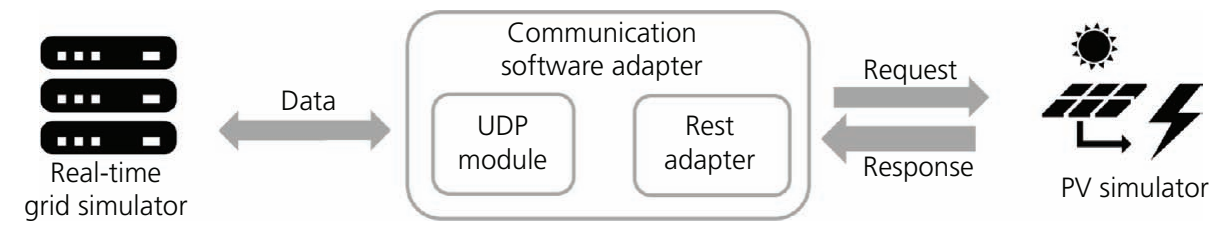

Figure 2. Integration of PV and real-time grid simulators. UDP, user datagram protocol 
Engineering Sustainability

Volume 172 Issue ES6
Planning and real-time management

of smart grids with high PV penetration

in Italy

Bottaccioli, Estebsari, Patti, Pons and Acquaviva

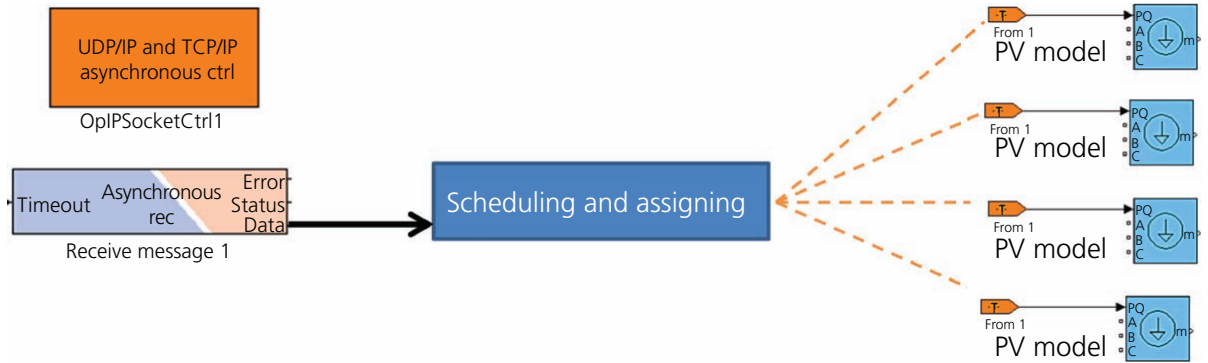

Figure 3. Real-time simulator receiving data links to the distributed PV models over the grid. IP, internet protocol; TCP, transmission control protocol

\subsection{Smart-grid simulation tool}

As mentioned in Section 2.1, the integration module of the PV simulator is in charge of correlating information coming from the smart grid with the estimated PV energy production for the same geographic area. This module also enables the communication with the real-time grid simulator through the communication software adapter (see Figure 2).

The communication software adapter has been developed following a methodology presented by Bottaccioli et al. (2017b). In its core, the communication software adapter consists of two submodules: (a) user datagram protocol (UDP) module and (b) Rest adapter. The UDP module interacts with the real-time simulation engine through a UDP server-client system: the UDP server receives and processes the information coming from the simulator, while the UDP client feeds the input data into the simulator. The Rest adapter parses the requests from the simulation engine and translates them into Rest calls to remote web services provided by the PV simulator. Finally, the web services' response is retrieved by the communication software adapter and its data are pushed into the real-time grid simulator again through the UDP module.

Considering the integrated simulation tool as a realised SIL, the environment which is the smart grid in the authors' case, is executed on the real-time simulator. The grid real-time simulation module is responsible for emulating the real grid behaviour facing different load or generation profile values and provides the status of the electrical system in terms of power flows, voltage profile and so on. The signals coming from outside of the real-time simulator control or define modelled prosumers' behaviour by updating PV generation output. During the simulation, the grid model requests the necessary values (active and reactive powers) to update modelled PV generation output and receives the required data from the PV simulator through appropriate UDP blocks inside the realtime model (Figure 3). The grid model can be run for an electromagnetic transient simulation with 50 to $250 \mu \mathrm{s}$ (or phasor



(a)

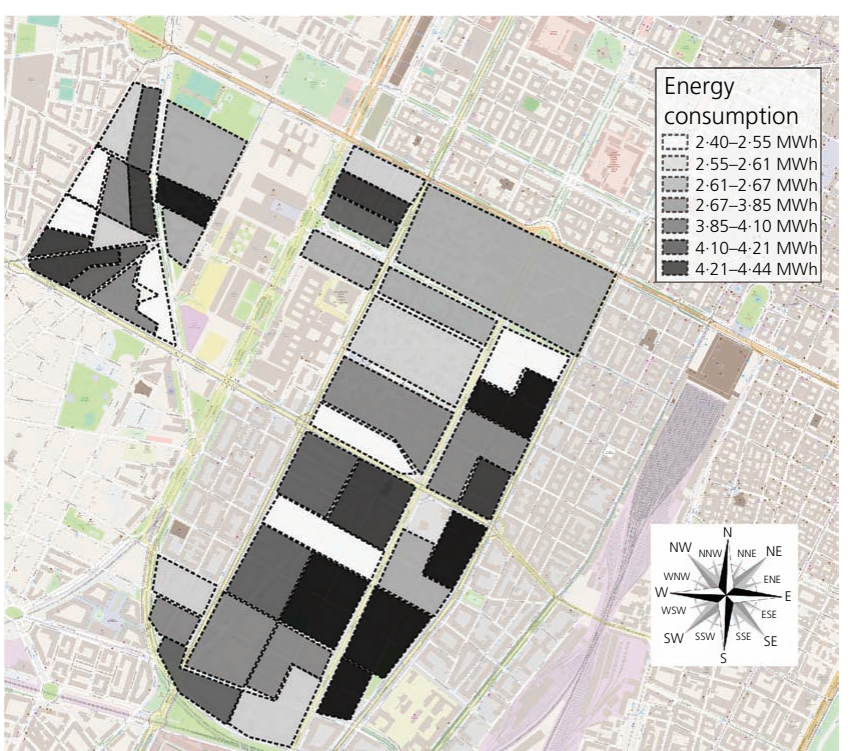

(b)

Figure 4. Diagram of case study area: (a) substation areas with buildings; (b) substation daily energy consumption. LV, low voltage; $\mathrm{MV}$, medium voltage 
Planning and real-time management of smart grids with high PV penetration in Italy

Bottaccioli, Estebsari, Patti, Pons and Acquaviva simulation with a few milliseconds) time steps, while the new values of PV generation can be updated every $15 \mathrm{~min}$.

The model is built with the SimPowerSystem (SPS) toolbox of Matlab Simulink. The Artemis software from Opal-RT is used to provide a fixed-step solver dedicated to complex power systems. It is an add-in toolbox to SPS enabling hard real-time simulation of power systems. The main modelled components of the distribution grid are a three-phase voltage source in series with a resistanceinductance (RL) branch as an equivalent model for the upstream high-voltage (HV) grid connected to a slack bus, three-phase twowinding transformers, three-phase $\pi$ section lines to model medium-voltage (MV) lines and three-phase three-wire dynamic load models with external control of active and reactive powers to model the prosumers. The prosumers in the present case study are (mainly) residential household as customers with their PV arrays on top of the building roofs as distributed producers. The net active and reactive powers of the prosumers are considered positive when the prosumer generation is lower than consumption.

\section{Case study and results}

The proposed methodology has been tested using information about Turin, which is a city in north-western Italy. The case study area involves a district where the DSM, with a resolution of $0.5 \mathrm{~m}^{2}$, and the MV grid topology are available. This area counts 2198 residential buildings connected to 43 medium-voltage/low-voltage (MV/LV) substations. Each substation serves an area whose extension depends on the number of connected households, as reported in Figure 4(a). To evaluate the integration of PV systems in the district, a sunny and cloudly summer days have been simulated. During summer days, in Italy, the energy consumption of residential users is lower than winter days (Maggiore, 2012) because residential households do not usually have air-conditioning systems. On the other hand, during the winter season, heating systems circulation pumps run almost all day long. In addition, sunny days in summer produce more electricity from PV systems and this can be a critical situation for distribution grids. Figure 4(b) shows the daily energy consumption for each substation (consumption data were obtained through measurements in $\mathrm{MV} / \mathrm{LV}$ substations). Energy consumption is not proportional to the area served by the substation, but rather to the number of households. Unfortunately, information about DNI and DHI are not available. Hence, the solar radiation decomposition module (see Figure 1) is used and the values of GHI are retrieved, through web services, from a weather station located in the centre of the district. Finally, high-quality monocrystalline silicon ( $\mathrm{Si}$ ) PV modules with efficiency $\eta_{\mathrm{pv}}=$ $20.4 \%$ in standard test conditions and thermal coefficient of maximum power $\gamma_{p v}=-0 \cdot 38 \% /{ }^{\circ} \mathrm{C}$ have been considered.

The electricity grid is an MV network with five feeders derived from three $22 \mathrm{kV}$ busbars of a $220 / 22 \mathrm{kV}$ primary substation. Each of the busbars is energised by a $220 / 22 \mathrm{kV}$ transformer. The total length of MV lines, mostly constituted by underground cables, is around $39 \mathrm{~km}$ (Figure 5). There are $49 \mathrm{MV} / \mathrm{LV}$ substations, out of which 43 supply loads (mainly 2200 residential buildings). The

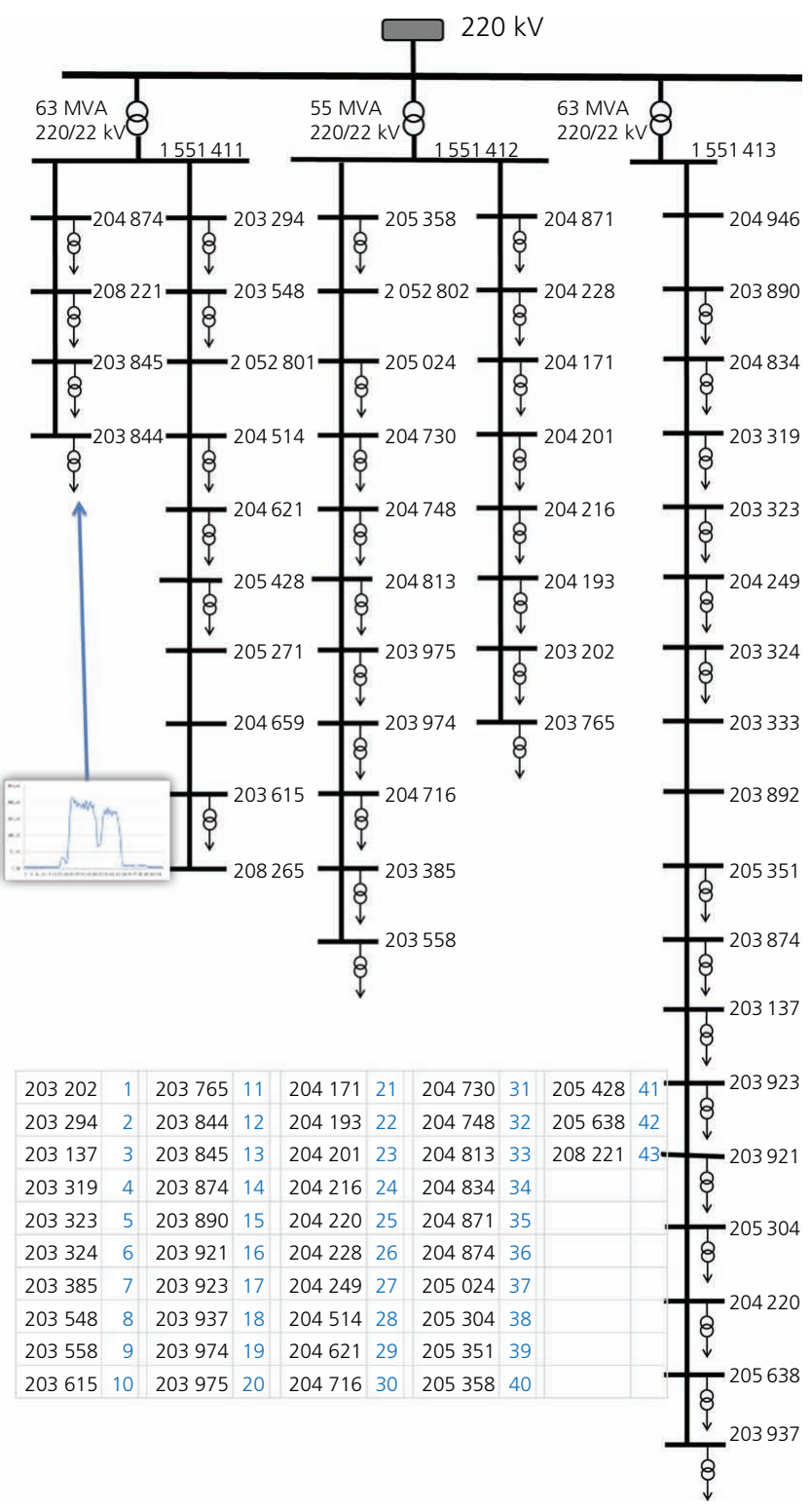

Figure 5. Case study electricity grid

$\mathrm{MV} / \mathrm{LV}$ transformers are characterised by voltage ratio of $22 \mathrm{kV} /$ $400 \mathrm{~V}$ and a nominal power of 400,250 or $160 \mathrm{kVA}$.

The authors use the integrated co-simulation platform to assess and discuss the impacts of rooftop PV penetration in this urban district on grid operation status from different perspectives. The advantages of this tool can be seen in both planning and operation.

In the phase of planning and network reinforcement, the tool can be used to assess the impacts of different levels of PV penetration on the existing grid. For example, the authors will show that problems can involve the transformers' capacity in the case of high penetration of PV in low-voltage grids. As another example of planning, homogeneous distribution of PV generation with respect 
Engineering Sustainability

Volume 172 Issue ES6
Planning and real-time management

of smart grids with high PV penetration

in Italy

Bottaccioli, Estebsari, Patti, Pons and Acquaviva to the level of demand should be taken into account. In the authors' simulation, it will be also shown how neglecting this point can introduce challenges in the network voltage control. Based on these studies, the existing network can be reinforced through investment or new regulation can be adjusted to meet the requirement of the system (e.g. when new regulations are being made to provide incentives to install PV arrays on the rooftop, different areas may get different incentives). Furthermore, impacts on the network could be mitigated by exploiting different tilt angles and orientations of PV systems, as reported by Freitas et al. (2018b). However, the objective of this work consists of maximising the energy production and evaluating the effect on the grid.

In the operation phase, the co-simulation tool proves how the PV simulator can provide in time quite sufficient information about generation to support low-voltage system state estimation (Pau et al., 2016). Since there are too many low-voltage connected PV panels in the grid, there are a lot of challenges in the estimation of generation data due to the need to install new suitable smart meters, acquiring a huge number of measurements, retrieving so much data so frequently (e.g. every $15 \mathrm{~min}$ ) and fast data processing. In the authors' simulation, it will be shown how a system operator can forecast and monitor substation power flow by using the data generated by the PV simulator.

In the district under analysis (see Section 3), among all the building rooftops, the suitable area module (see Figure 1) identified 944 areas, equivalent to $71595.53 \mathrm{~m}^{2}$, suitable for deploying PV systems with a nominal power potential equal to 14.21 MW. The distribution of power and energy production for each MV substation area during a sunny day in summer is shown in Figures 6(a) and 6(b), respectively. As the simulation process also considers the shadows of surrounding buildings and vegetation, rooftop areas with high power production potential can have an energy production impact lower than areas with low power production potential (see Figures 6(a) and 6(b)). During the sunny day, the peak power production is around $3.77 \mathrm{MW}$ and the energy production is equal to $28.41 \mathrm{MWh}$. On the other hand, during the cloudy day, it is around $3.94 \mathrm{MW}$ with an energy production of $16.95 \mathrm{MWh}$. The peak power production does not reach the nominal peak power in either of the two cases. The higher peak power reached on the cloudy day can be explained through the phenomenon of irradiance spikes caused by broken clouds (Chicco et al., 2015) or by a lower temperature of the PV arrays.

The authors discuss in the next paragraphs the results in the two aforementioned phases (i.e. planning and operation) for voltage control, transformers' capacity and substation power flow.

\subsection{Voltage profile}

In distribution systems, tap changers of the HV/MV transformers at the primary substations would try to keep the voltage at the MV busbars at a certain level, by measuring and monitoring transformer current. When a transformer feeds several feeders, characterised by different PV penetrations with respect to peak loads, the voltage profiles at the secondary substations on the different feeders follow different profiles. This means that monitoring and regulating the voltage at the beginning of feeders is not necessarily sufficient for keeping voltages of all the substations of all the lines in the desired range. For example, in feeders where generation is higher, in some substations voltages may be above the admissible limit. In the authors' case, the first feeder from the left is characterised by low demand, while its PVgenerated power is more or less the same as the others. In the second feeder, MV/LV transformers have greater sizes (400 and

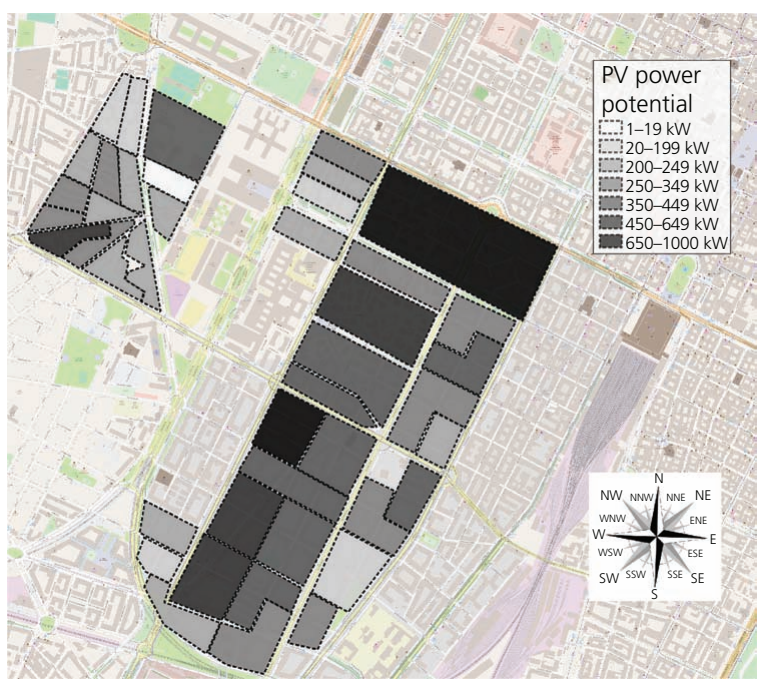

(a)

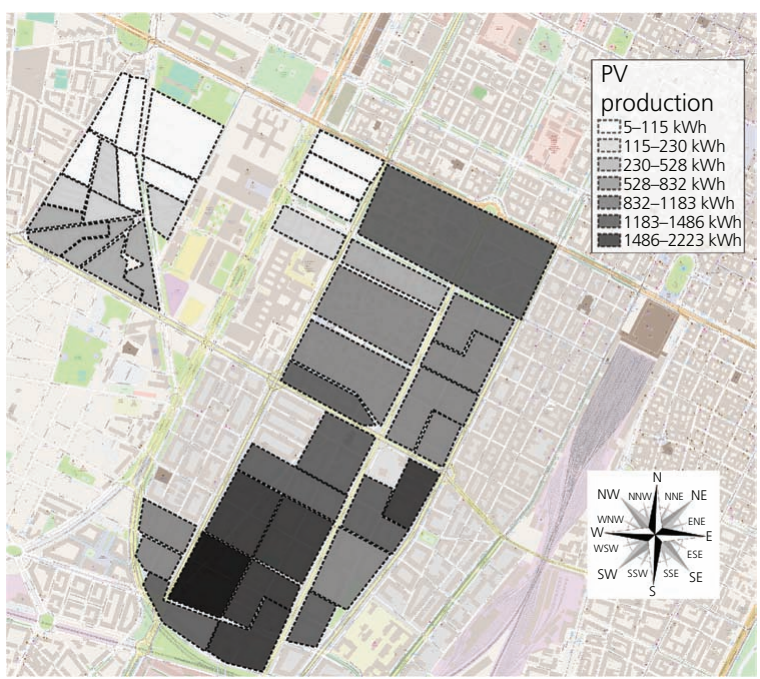

(b)

Figure 6. Diagram of PV potential and energy production: (a) PV power potential (sunny day); (b) total PV generation (sunny day) 
Planning and real-time management

of smart grids with high PV penetration

in Italy

Bottaccioli, Estebsari, Patti, Pons and Acquaviva
$250 \mathrm{kVA}$ ), and consumption is higher than in the left one in which smaller transformers $(160 \mathrm{kVA})$ are installed. The authors ran the co-simulation for a summer scenario of a sunny day. Figures 7 and 8 show aggregated generation and consumption at substations 7, 9, 21 and 24, and Figure 9 shows the net active power injected into the downstream LV grids connected to these substations. Substations 21 and 24 with high self-sufficiency are connected to one feeder, and substations 7 and 9 with lower self-sufficiency are connected to the other feeder. According to the voltage profiles of these substations (Figure 10), any changes in the level of transformer voltage to correct over-/under-voltage in one feeder would result in more deviation in the other feeder.

\subsection{Transformers" capacity}

The MV/LV transformers at the secondary substations are modelled based on the existing transformers in the real network, which were installed without considering the new PV generation capacity. The maximum aggregated generated power of each substation on the study day is shown in Figure 11(a).

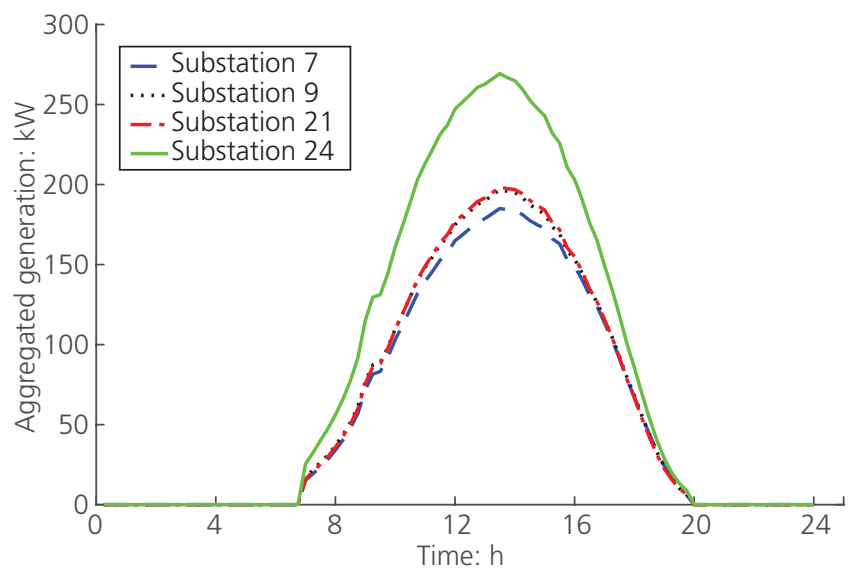

Figure 7. Aggregated PV generation in some substations

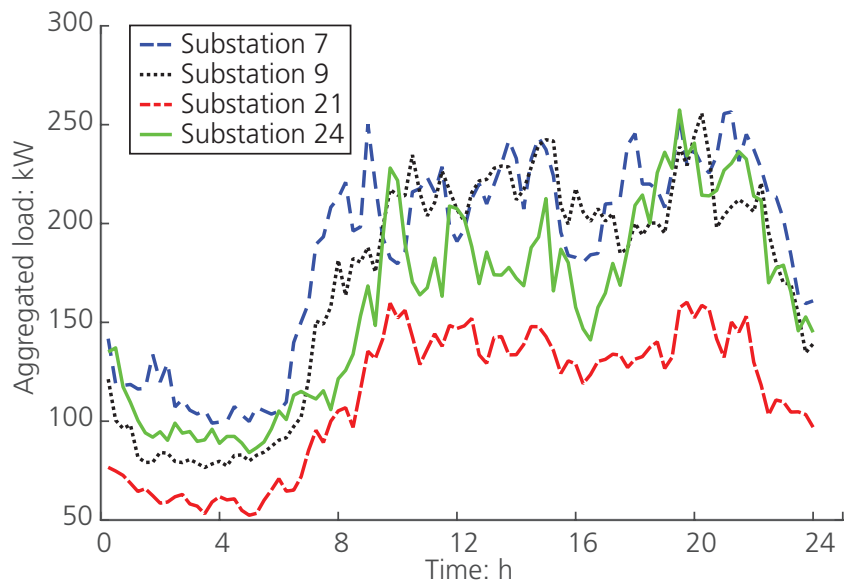

Figure 8. Aggregated load in some substations

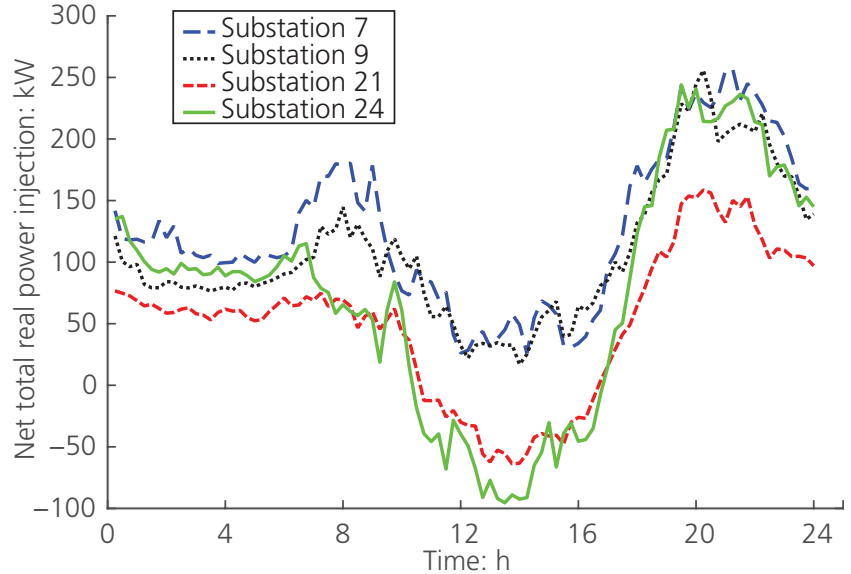

Figure 9. Net total real power injection

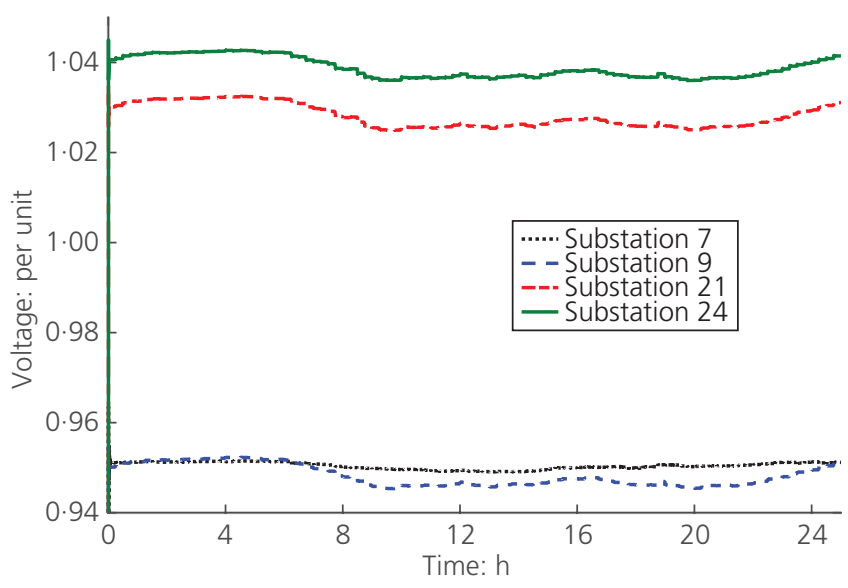

Figure 10. Voltage profiles of some substations

Transformer capacity is based on maximum apparent power in kilovolt-amperes and can be considered for both power absorption and injection (the specular positive and negative plot lines in Figure 12). All values of power generation and consumption are calculated for every $15 \mathrm{~min}$ of the study day; therefore, there would be 96 snapshots of the system status. To reach the worst scenario, the authors consider the maximum net consumption (subtracting local generation from local consumption) of each substation during the study day, indicated with large pale bars in Figure 12. As shown with narrow dark bars, the maximum load consumption of all substations is within the transformer capacity range, while integrating $\mathrm{PV}$ generation would cause violations in two substations (24 and 30 in Figure 12). The maximum net consumption in these two substations exceeds the transformers' capacity due to the high amount of PV generation and low consumption. This highlights the fact that in cases where local generation is much more than local demand, either the installation of PV arrays should be reduced or grid infrastructures in terms of transformers (and also cables/lines) should be enhanced to tolerate 


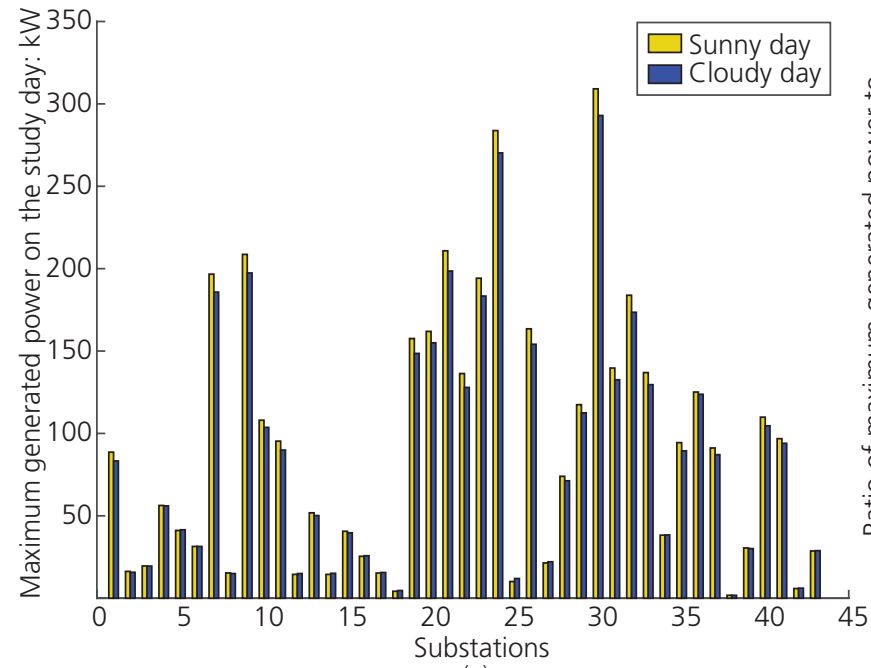

(a)

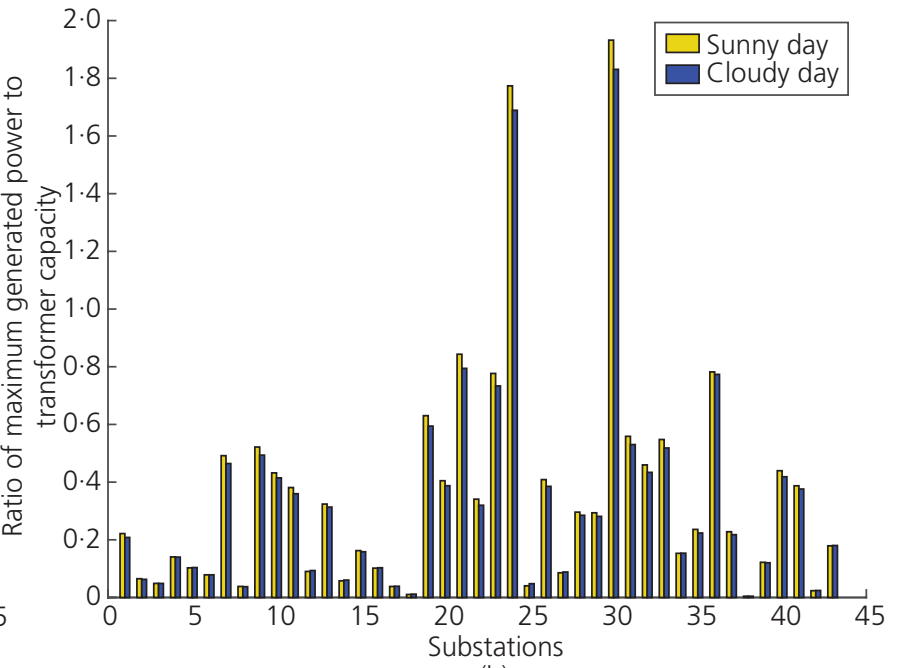

(b)

Figure 11. Maximum PV generation of each MV/LV substation and transformer's capacity: (a) maximum generated power in a sunny or cloudy day; (b) ratio between maximum generated power and transformer capacity in (a)



Figure 12. Transformer capacity results

reverse power injection from substations to the grid. Another possible approach consists of installing a PV system with different tilt angles and orientations to match power production of PV systems with power consumption. This reduces the power injected in the grid but also reduces the maximum power that can be potentially produced by PV systems. Hence, an accurate multicriteria cost-benefit analysis should be performed to evaluate the best option.

\subsection{Power flow}

In this case study, the authors analyse a scenario in which almost all residential buildings install PV arrays on their rooftops, which reach their peak power on a sunny day of July. The PV generation module does not consider grid constraints and introduces PV generation with the highest possible penetration, considering the available surface areas on the rooftops. In the present case study, the penetration level is $51 \%$, where the PV penetration level is defined as the total peak PV generation divided by the total peak load apparent power. The total PV generation profile in this area in a cloudy day during July is shown in Figure 13 with a solid curve. An effect of high PV penetration on such a cloudy day can be observed when generation profiles change dramatically within a few seconds. If a huge generation drop occurs exactly in the ascending period of consumption profile, in high-PV-penetration scenarios, a large power 
Planning and real-time management

of smart grids with high PV penetration

in Italy

Bottaccioli, Estebsari, Patti, Pons and Acquaviva

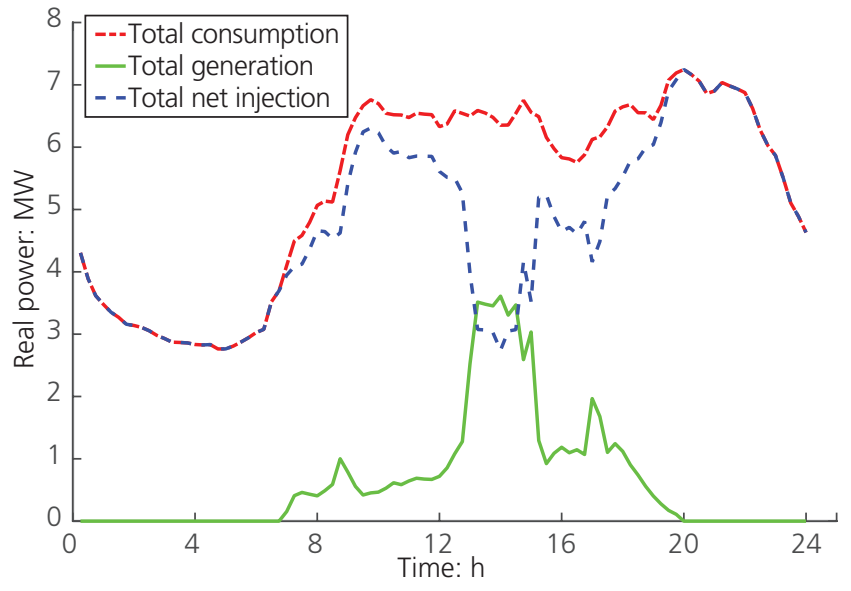

Figure 13. Total load, generation and net real power

deficit will be experienced. The short dashed curve shows the result of power flow at the primary substation in terms of total active power injected into the distribution system; the steep descents mean rapid demand reduction in the distribution system $(1.7 \mathrm{MW})$, and the steep rises imply a fast demand increase in the distribution system. Thanks to the PV simulator, the distribution management system (DMS) can perform some analysis in advance (e.g. 15 min earlier) to prevent these steeps - for example, by demand-side management through flexible loads or PV curtailment.

The added value of this integrated framework is the possibility of concurrently taking into accounts both real-like PV generation behaviour from one side and grid behaviour and constraints on the other side, highlighting the fact that it can support the DMS during operation mode.

\section{Conclusion}

In this paper, the authors present an integrated real-time platform for the assessment of the impacts of PV DG in smart grids. The platform comprises two main components, a PV simulator and a real-time distribution network simulator. The two components are interconnected over the internet through a communication software adapter. The proposed tool allows for the simulation of the power produced by PV generators on building rooftops under real-sky conditions and for the analysis of the distribution grid behaviour in this DG scenario. The tests carried out on a case study based on a district of Turin, Italy, show that the distribution network, in the actual configuration, may not be ready to accommodate all the generation capacity that can be installed if all the available rooftop surface is exploited, as, for example, in certain hours of the day and on certain days of the year the capacity of some transformers is exceeded. The presented platform could be useful, in the future, to analyse different penetration scenarios, to test new operational procedures for the distribution network and to verify the impact of new connection rules and new regulations. Furthermore, the presented platform could be coupled and integrated with energy consumption estimation tools such as those described by Gruber et al. (2017) if measured load profiles are missing. In the case study presented in this paper, the behaviour of the LV grid is neglected and all the prosumers fed by a substation are studied in an aggregated way. This is due to two main problems: usually it is not easy to obtain detailed and precise information on the LV distribution system and considering LV feeders as well would make the simulation complexity too high. In this case study, to estimate the energy production of PV systems, real-sky conditions have been simulated by applying some models in the literature to decompose global horizontal irradiation into direct and diffuse irradiation. This can be considered a limitation of the PV simulator because the final error rate increases. However, the PV simulator is ready to exploit also real measurements sampled by sensors for direct and diffuse irradiation. This bypasses the decomposition models and decreases the error rate. However, these sensors are not installed widely in common weather stations because of their high cost.

\section{Acknowledgements}

This work was partially supported by the EU projects district information modeling and management for energy reduction (Dimmer) and flexible smart metering for multiple energy vectors with active prosumers (Flexmeter) and by the Italian project 'Edifici a Zero Consumo Energetico in Distretti Urbani Intelligenti'.

\section{REFERENCES}

Bompard E, Monti A, Tenconi A et al. (2016) A multi-site real-time co-simulation platform for the testing of control strategies of distributed storage and V2G in distribution networks. Proceedings of the 18th 2016 European Conference on Power Electronics and Applications, 2016 (EPE'16 ECCE), Karlsruhe, Germany.

Bottaccioli L, Patti E, Acquaviva A et al. (2015) A tool-chain to foster a new business model for photovoltaic systems integration exploiting an energy community approach. Proceedings of the 20th Conference on Emerging Technologies \& Factory Automation (ETFA), Luxembourg, Luxembourg.

Bottaccioli L, Patti E, Grosso M et al. (2016) Distributed software infrastructure for evaluating the integration of photovoltaic systems in urban districts. Proceedings of the 5th International Conference on Smart Cities and Green ICT Systems (SMARTGREENS), Rome, Italy, pp. 357-362.

Bottaccioli L, Patti E, Macii E and Acquaviva A (2017a) GIS-based software infrastructure to model PV generation in fine-grained spatiotemporal domain. IEEE Systems Journal 12(3): 2832-2841, https://doi. org/10.1109/JSYST.2017.2726350.

Bottaccioli L, Estebsari A, Pons E et al. (2017b) A flexible distributed infrastructure for real-time co-simulations in smart grids. IEEE Transactions on Industrial Informatics 13(6): 3265-3274, https://doi. org/10.1109/TII.2017.2702206.

Bottaccioli L, Estebsari A, Patti E, Pons E and Acquaviva A (2017c) A novel integrated real-time simulation platform for assessing photovoltaic penetration impacts in smart grids. Energy Procedia 111: 780-789, https://doi.org/10.1016/j.egypro.2017.03.240.

Brihmat F and Mekhtoub S (2013) PV cell temperature/PV power output relationships homer methodology calculation. Proceedings of the Conférence Internationale des Energies Renouvelables, CIER'13, Sousse, Tunisia.

Brito M, Freitas S, Guimarães S, Catita C and Redweik P (2017) The importance of facades for the solar PV potential of a Mediterranean city using LiDAR data. Renewable Energy 111: 85-94, https://doi.org/ 10.1016/j.renene.2017.03.085. 
Engineering Sustainability

Volume 172 Issue ES6
Planning and real-time management

of smart grids with high PV penetration

in Italy

Bottaccioli, Estebsari, Patti, Pons and Acquaviva
Camargo LR, Zink R, Dorner W and Stoeglehner G (2015) Spatio-temporal modeling of roof-top photovoltaic panels for improved technical potential assessment and electricity peak load offsetting at the municipal scale. Computers, Environment and Urban Systems 52: 58-69, https://doi.org/10.1016/j.compenvurbsys.2015.03.002.

Chicco G, Cocina V, Di Leo P, Spertino F and Massi Pavan A (2015) Error assessment of solar irradiance forecasts and AC power from energy conversion model in grid-connected photovoltaic systems. Energies 9(1): 8, https://doi.org/10.3390/en9010008.

De Amicis R, Conti G, Patti D, Ford M and Elisei P (2012) Proceedings of the 17th International Conference on Urban Planning and Regional Development in the Information Society GeoMultimedia 2012 (REAL CORP 2012), Schwechat, Australia, pp. 14-16.

de Sousa L, Eykamp C, Leopold U, Baume O and Braun C (2012) iGuess - a web based system integrating urban energy planning and assessment modelling for multi-scale spatial decision making. In International Environmental Modelling and Software Society (iEMSs) 2012 International Congress on Environmental Modelling and Software. Managing Resources of a Limited Planet: Pathways and Visions under Uncertainty, Sixth Biennial Meeting, Leipzig, Germany (Seppelt R, Voinov AA, Lange S and Bankamp D (eds)). International Environmental Modelling and Software Society, Manno, Switzerland, pp. 171-178.

Domínguez J and Amador J (2007) Geographical information systems applied in the field of renewable energy sources. Computers \& Industrial Engineering 52(3): 322-326, https://doi.org/10.1016/j.cie.2006.12.008.

Dufour C, Blanchette H and Bélanger J (2008) Very-high speed control of an FPGA-based finite-element-analysis permanent magnet synchronous virtual motor drive system. Proceedings of the 2008 34th Annual Conference of IEEE Industrial Electronics, Orlando, FL, USA, pp. 2411-2416.

Engerer N (2015) Minute resolution estimates of the diffuse fraction of global irradiance for southeastern Australia. Solar Energy 116: 215-237, https://doi.org/10.1016/j.solener.2015.04.012.

Estebsari A, Pons E, Patti E et al. (2016) An IoT realization in an interdepartmental real time simulation lab for distribution system control and management studies. Proceedings of the 2016 IEEE 16th International Conference on Environment and Electrical Engineering (EEEIC), Florence, Italy.

Fielding RT and Taylor RN (2002) Principled design of the modern web architecture. ACM Transactions on Internet Technology 2(2): 115-150, https://doi.org/10.1145/514183.514185.

Fowler M and Lewis J (2014) Microservices. Martin Fowler, Chicago, IL, USA. See http://martinfowler.com/articles/microservices.html (accessed 08/09/2018).

Freitas S, Catita C, Redweik P and Brito M (2015) Modelling solar potential in the urban environment: state-of-the-art review. Renewable and Sustainable Energy Reviews 41: 915-931, https://doi.org/10.1016/ j.rser.2014.08.060

Freitas S, Santos T and Brito MC (2018a) Impact of large scale PV deployment in the sizing of urban distribution transformers. Renewable Energy 119: 767-776, https://doi.org/10.1016/j.renene.2017.10.096.

Freitas S, Reinhart C and Brito MC (2018b) Minimizing storage needs for large scale photovoltaics in the urban environment. Solar Energy 159: 375-389, https://doi.org/10.1016/j.solener.2017.11.011.

Gagliano A, Nocera F, Detommaso M and Spataru C (2017) A geographic information method for managing urban energy use. Proceedings of the Institution of Civil Engineers - Engineering Sustainability 170(1): 19-32, https://doi.org/10.1680/jensu.15.00052.

Gruber JK, Prodanovic M and Alonso R (2017) Estimation and sensitivity analysis of building energy demand. Proceedings of the Institution of Civil Engineers - Engineering Sustainability 170(2): 81-92, https://doi.org/10.1680/jensu.15.00049.

Hofierka J and Kaňuk J (2009) Assessment of photovoltaic potential in urban areas using open-source solar radiation tools. Renewable Energy 34(10): 2206-2214, https://doi.org/10.1016/j.renene.2009.02.021.
Jakubiec JA and Reinhart CF (2013) A method for predicting city-wide electricity gains from photovoltaic panels based on LiDAR and GIS data combined with hourly Daysim simulations. Solar Energy 93: 127-143, https://doi.org/10.1016/j.solener.2013.03.022.

Karatasou S, Santamouris M and Geros V (2003) Analysis of experimental data on diffuse solar radiation in Athens, Greece, for building applications International Journal of Sustainable Energy 23(1-2): 1-11, https://doi. org/10.1080/0142591031000148597.

Keane A, Ochoa LF, Borges CL et al. (2013) State-of-the-art techniques and challenges ahead for distributed generation planning and optimization. IEEE Transactions on Power Systems 28(2): 1493-1502, https://doi.org/10.1109/TPWRS.2012.2214406.

Linke F (1922) Transmissions-koeffizient und trübungsfaktor. Beiträge zur Physik der Atmosphäre 10: 91-103 (in German).

Luka N, Seme S, Žlaus D, Štumberger G and Žalik B (2014) Buildings roofs photovoltaic potential assessment based on LiDAR (Light Detection and Ranging) data. Energy 66: 598-609, https://doi.org/10. 1016/j.energy.2013.12.066.

Maggiore S (2012) Analisi di impatto dellintroduzione della tariffa bioraria obbligatoria. Ricerca sul Sistema Energetico, Milan, Italy. See http://doc.rse-web.it/doc/doc-sfoglia/13000580-315241/13000580315241.html (accessed 10/05/2016) (in Italian).

Mapdwell (2018) http://www.mapdwell.com (accessed 15/07/2018).

Newman S (2015) Building Microservices. O’Reilly Media, Inc., Sebastopol, CA, USA.

Pak LF and Dinavahi V (2009) Real-time simulation of a wind energy system based on the doubly-fed induction generator. IEEE Transactions on Power Systems 24(3): 1301-1309, https://doi.org/10. 1109/TPWRS.2009.2021200.

Park M and Yu IK (2004) A novel real-time simulation technique of photovoltaic generation systems using RTDS. IEEE Transactions on Energy Conversion 19(1): 164-169, https://doi.org/10.1109/TEC.2003. 821837.

Patti E, Pons E, Martellacci D et al. (2015) multiFLEX: flexible multiutility, multi-service smart metering architecture for energy vectors with active prosumers. Proceedings of the 4th International Conference on Smart Cities and Green ICT Systems (SMARTGREENS), Lisbon, Portugal, pp. 288-293.

Patti E, Syrri ALA, Jahn M et al. (2016) Distributed software infrastructure for general purpose services in smart grid. IEEE Transactions on Smart Grid 7(2): 156-1163, https://doi.org/10.1109/TSG.2014. 2375197.

Pau M, Patti E, Barbierato L et al. (2016) Low voltage system state estimation based on smart metering infrastructure. Proceedings of the 2016 IEEE International Workshop on Applied Measurements for Power Systems (AMPS), Aachen, Germany.

Pau M, Patti E, Barbierato L et al. (2018) A cloud-based smart metering infrastructure for distribution grid services and automation. Sustainable Energy, Grids and Networks 15: 14-25, https://doi.org/10. 1016/j.segan.2017.08.001.

Ruiz-Arias J, Alsamamra H, Tovar-Pescador J and Pozo-Vázquez D (2010) Proposal of a regressive model for the hourly diffuse solar radiation under all sky conditions. Energy Conversion and Management 51(5): 881-893, https://doi.org/10.1016/j.enconman.2009.11.024.

Spertino F, Di Leo P and Cocina V (2014) Which are the constraints to the photovoltaic grid-parity in the main European markets? Solar Energy 105: 390-400, https://doi.org/10.1016/j.solener.2014.03.021.

Suri M, Huld T, Dunlop E and Cebecauer T (2008) Geographic aspects of photovoltaics in Europe: contribution of the PVGIS website. IEEE Journal of Selected Topics in Applied Earth Observations and Remote Sensing 1(1): 34-41, https://doi.org/10.1109/JSTARS.2008.2001431.

Vamsidhar S and Fernandes B (2004) Hardware-in-the-loop simulation based design and experimental evaluation of DTC strategies. Proceedings of the 2004 IEEE 35th Annual Power Electronics Specialists Conference, Aachen, Germany, vol. 5, pp. 3615-3621. 
Engineering Sustainability

Volume 172 Issue ES6
Planning and real-time management

of smart grids with high PV penetration

in Italy

Bottaccioli, Estebsari, Patti, Pons and Acquaviva
Varney KE and Vahdati MM (2014) Simulations of a photovoltaic-thermal ground source heat pump system. Proceedings of the Institution of Civil Engineers - Engineering Sustainability 168(1): 28-37, https:// doi.org/10.1680/ensu.14.00013.

Wang G and Gao W (2008) Real time simulation for wind power generation system using RTDS. Proceedings of the 2008 40th North American Power Symposium, Calgary, AB, Canada.
Weather Underground (2017) http://www.wunderground.com (accessed 18/10/2017).

Yu JS, Kim JH, Kim SM and Kim JT (2017) Thermal and energy performance of a building with PV-applied double-skin façade. Proceedings of the Institution of Civil Engineers - Engineering Sustainability 170(6): 345-353, https://doi.org/10.1680/jensu.16. 00017.

\section{How can you contribute?}

To discuss this paper, please email up to 500 words to the editor at journals@ice.org.uk. Your contribution will be forwarded to the author(s) for a reply and, if considered appropriate by the editorial board, it will be published as discussion in a future issue of the journal.

Proceedings journals rely entirely on contributions from the civil engineering profession (and allied disciplines). Information about how to submit your paper online is available at www.icevirtuallibrary.com/page/authors, where you will also find detailed author guidelines. 